\title{
Effects of Alterations in Staphylococcus aureus Cell Membrane and Cell Wall in Antimicrobial Resistance
}

\author{
Andrea de Souza Monteiro, \\ Wallace Ribeiro Nunes Neto, \\ Aleff Ricardo Santos Mendes, \\ Bruna Lorrana dos Santos Pinto, \\ Luís Cláudio Nascimento da Silva and \\ Gabriella Freitas Ferreira
}

Additional information is available at the end of the chapter

http://dx.doi.org/10.5772/66954

\begin{abstract}
Staphylococcus aureus is one of the most successful opportunistic pathogen able to cause serious infections due to its ability to produce virulence factors and acquire antimicrobial resistance. Recent reports indicate that the phenotypic changes in the cell wall and cell membrane are essential mechanisms related to the resistance to several antibacterial drugs (such as daptomycin and vancomycin). These alterations involve changes in cell wall composition and chemical modifications of some components (point mutation leading to modification in phosphatidylglycerol molecule, in the production of the aberrations in peptidoglycan structure and decrease in autolytic activity of the components of the cell envelope), leading to changes in electric charge of the cell surface, cell membrane fluidity and cell morphology. In fact, S. aureus develops several multifactorial and strainspecific adaptive mechanisms to survival in host. The study of such mechanisms is very important. The aim of this chapter is to review the phenotypic mechanisms related to drug resistance in S. aureus.
\end{abstract}

Keywords: Staphylococcus aureus, daptomycin, vancomycin, heteroresistance, stress stimulon response

\section{Introduction}

The lipopeptide and glycopeptide antibiotics are very effective against infections caused by Gram-positive cocci, showing good efficacy against Staphylococcus aureus. The mechanism of 
action of these antibiotics is linked with their ability to block cell wall bacterial synthesis. As examples of the most used drugs to treat $S$. aureus infection, we have daptomycin (DAP) and vancomycin. However, currently, the indiscriminate use of these antimicrobials has decreased their effectiveness against $S$. aureus strains [1].

Daptomycin (DAP) is a cyclic lipopeptide antibiotic obtained from Streptomyces roseosporus with activity against the most Gram-positive bacteria, including vancomycin-resistant enterococci (VRE) and methicillin-resistant S. aureus (MRSA) [2-4]. DAP appears to have multiple effects on the Gram-positive bacterial cell membrane (BCM) [5]. The mechanism of action proposed is the aggregation between DAP and BMC, which alters the architecture of cell membrane and forms pores, leading to ion loss. This new arrangement leads to a rapid depolarization, resulting in a loss of membrane potential that culminates in bacterial cell death. Regarding the interaction mechanisms with the formation of cell wall and surface, a special mention should be given to the interference in peptidoglycan and lipoteichoic biosynthesis [6-8].

The glycopeptide antibiotics were introduced in therapy against infections caused by S. aureus in 1955 [9]. The vancomycin is glycopeptide produced by Amycolatopsis orientalis (actinomycete) and is the representative of this class of antibiotic widely used in medical practice today, especially after the appearance of resistant strains of $S$. aureus to methicillin [10]. However, the emergence of molecular mechanisms of resistance to vancomycin and the appearance of phenotypic resistance profiles in heterogeneous subpopulations of $S$. aureus as described susceptible have hindered the use of glycopeptide as first choice agent in antibiotic therapy [11]. Some reduced susceptibility phenotypes are classified in some profiles as vancomycin-intermediate S. aureus (VISA) and vancomycin-resistant S. aureus (VRSA) [11]. Subpopulations of heterogeneous $S$. aureus with heterogeneous resistance to vancomycin and notoriously sensitive to vancomycin are classified as heteroresistant vancomycin-intermediate S. aureus (hVISA) [1].

Heteroresistance is defined as resistance to antibiotics expressed by a subset of a microbial population that initially is considered susceptible to these antibiotics. These resistant subpopulations are able to adapt to increasing drug concentrations in a stepwise manner [12]. This phenomenon has been described in a wide range of bacteria and fungi, but attention has been directed toward S. aureus [13]. For the S. aureus hVISA strains containing subpopulations of vancomycin-intermediate daughter cells, the minimal inhibitory concentrations (MICs) for the parent strains of these daughter cells fall within the susceptible range of $1-4 \mu \mathrm{g} / \mathrm{mL}$. However, if the lineage is exposed to increasing concentrations of vancomycin, a new generation of cells with reduced susceptibility profiles will be favored. This exhibition creates a selective pressure that favors the outgrowth of rare vancomycin-resistant clones leading to hVISA clones. Eventually, the continued exposure to vancomycin culminates in a uniform population of VISA phenotypes [14]. Evidence in clinical case studies which monitored S. aureus infections in 250 patients showed that the hVISA phenotype is closely related with significantly prolonged bacteremia events and associated with increased rates of endocarditis and osteomyelitis, compared with methicillin-resistant Staphylococcus aureus (MRSA) bacteremia [15].

VISA and hVISA have an increased D-Ala-D-Ala moieties in their thickness cell wall that form false targets that sequester vancomycin, with reduced autolytic activity and slow growth in 
vitro ([14]. This phenomenon is believed to "trap" vancomycin and this, together with the thickened cell wall, may act as a barrier to the diffusion of large molecules, like daptomycin (as cited below) $[16,17]$. In this way, the emergence of these bacterial phenotypes cited above has put into question the effectiveness of these antibiotics against $S$. aureus $[1,18,19]$. These changes in susceptibility seem to be related to the difficulty of resolving cases of $S$. aureus infection, leading to increased mortality of patients [20]. Altered host-pathogen interaction due to hVISA strains may change the course of the infection to worst prognostic for the patient. In a macrophage model of infection, hVISA/VISA strains showed increased capsule and reduced protein A (SpA, a protein surface cell), associated with reduced NF- $\kappa B$ activation and reduced TNF- $\alpha$ and IL-1 $\beta$ expression [21]. The persistent infections associated with hVISA/VISA strains can be a result of changes in host-pathogen interactions that culminate in attenuated host immune response [14].

\section{Mechanisms for daptomycin resistance associated to modifications in cell membrane of the Staphylococcus aureus}

A few years ago, antibiotic therapy using DAP to treat MRSA infections appeared to be a good choice; however, there are reports in the literature about bacterial strains with resistance to daptomycin (DAP-R) and with intermediary resistance (DAP-I) [19, 22]. S. aureus DAP-R infection has been associated with endocarditis and abscesses; it is characterized by high concentration of microorganisms in infected area with oral administration of low doses of DAP (i.e., $\leq 6 \mathrm{mg} / \mathrm{kg} /$ day) $[23,24]$. In addition, it was observed the emergence of DAP non-susceptible $S$. aureus phenotypes in a patient with persistent VISA bacteremia [1]. The resistant phenotypes of VISA strains have developed an increased DAP resistance during therapy [25, 26]. Besides, it could be associated with the previous use of vancomycin [27]. DAP-I mechanisms in $S$. aureus have not yet been elucidated completely, but a possible predominant phenomenon for the appearance of this phenotype would be an increase repulsion of DAP molecules of the surface cell. This modification is generally associated with an overall net charge change on the cell surface (for a more positive charge) [23].

The lysyl-phosphatidylglycerol (Lys-PG) is an important membrane lipid in bacteria, more common in Gram-positive cells [28]. The Lys-PG is incorporated into the microbial cell membrane by the activity of the multiple peptide resistance factor $(\mathrm{MprF})$ gene product (encoded by the $m p r F / f m t C$ gene). The $\mathrm{MprF}$ is a bifunctional protein composed by C-terminal part responsible by synthesize of the Lys-PG and the N-terminal hydrophobic protein domain is essential for efficient translocation of Lys-PG from the inner to the outer leaflet of the cytoplasmic membrane bacteria [28, 29].

The MprF protein catalyzes the modification of the negatively charged in phosphatidylglycerol (PG) with l-lysine and translocation of Lys-PG from the inside to the outside of the cell membrane leaflet [30]. MprF proteins in S. aureus encompass a lysyl-phosphatidylglycerol synthase and a Lys-PG flippase domain, responsible for aiding the movement of phospholipid molecules between the two leaflets in cell membrane ("flip-flop" transition). Moreover, in S. aureus 
the Lys-PG flippase and synthase in the MprF can understand two different proteins fused with distinct functional subunits [29].

The neutralization of the cell membrane surface leads to MprF triggered by bacterial resistance to cationic antimicrobial peptides (CAMPs) [31]. MprF was first described as a virulence factor of $S$. aureus by Peschel et al. [32], analyzing a mutant strain observed for MprF gene, which was unable to modify phosphatidylglycerol with L-lysine, was considerably faster killed by human neutrophils and exhibited an attenuated virulence in mice. This study indicated a key role of MprF for the resistance to defensins and cathelicidins (secreted peptides in host mucosal) and implied a higher pathogenicity of S. aureus. Interestingly, MprF is involved in the development of DAP-R in S. aureus [33]. These observations related to cells with DAP-R prolife take into account several factors. Among these are mechanisms that can interfere with the balance in incorporation of the PG into membrane. Also, it was verified that the co-expression of the alanyl-PG (Ala-PG) synthase with flippase domains of Lys-PG synthesizing MprF proteins led to a wild-type level of daptomycin susceptibility, indicating that Ala-PG can also protect bacterial membrane against DAP [33]. Moreover, the incorporation of the point mutations leading to amino acid exchanges in the MprF proteins of S. aureus strains leads to a decreased susceptibility to daptomycin [34].

Several hypotheses have been raised about the interference mechanisms of a mutant gene for the MprF protein in the dynamics of PG synthesis in S. aureus. It is considered that the biophysicist repulsion process of DAP molecules from the bacterial surface would be assigned to an increased PG incorporation by $\triangle m p r F$ strain with singly point-mutated $m p r F$ gene $\left(m p r F_{\mathrm{S}_{295 \mathrm{~L}}}\right.$ or $\left.m p r F_{\mathrm{T} 345 \mathrm{~A}}\right)$ [35]. More recently, it was noted that the decrease in susceptibility to DAP in some mutant strains for the MprF gene was the result of the critical effects caused by PG poor incorporation, resulting in failures in the oligomerization in the cell membrane, which compromise its antibacterial activity [23]. In addition, the possibility of antibiotic repulsion (such as DAP) triggered by the increase in positive charge can be seen in some $S$. aureus strains with a significant increase in cell wall thickness. These strains exhibit increased MICs for drug antibiotic and VISA-DAP-R phenotype [1].

\section{Mechanisms for vancomycin resistance associated to modifications in cell envelope of the Staphylococcus aureus}

The resistance mechanisms for both VISA and hVISA appear to have common features. However, it is always observed in patterns that distinguish them from vancomycin-susceptible Staphylococcus aureus (VSSA). Although the gene expression of hVISA or VISA during exposure to vancomycin denotes some patterns, subtle differences in the contribution of transcripts of these two phenotypes are found. One basic evidence regarding decrease in susceptibility is related to the cell wall thickening in bacterial cells. This process seems to initiate with the acquisition of a reduced autolysis caused by downregulation of autolysin genes, such as atl/lytM [1]. A thickened cell wall has been highlighted as a characteristic phenotype commonly found in clinical VISA. This feature is intimately associated with peptidoglycan-clogging theory that explains vancomycin resistance by passage delay of antibiotic molecules across the thickened 
peptidoglycan layers [18]. Punctual mutations by genetic manipulation in S. aureus strains that could lead to cell wall thickening were investigated thoroughly. However, these studies did not conclude that active point mutations involving genes of regulatory systems, autolysins and major catalytic enzymes could be involved in the appearance of VISA phenotypes [36, 37].

Studies conducted on gene transcription analysis showed that after exposure of DAP, the cells exhibit a regulation of the cell wall "stimulon," similar to that observed in response to vancomycin $[1,38]$. Comparison of DAP-I and DAP-S. aureus strain pairs, which are located in the genes that have been associated with VISA phenotype, indicated that the genes involved in the synthesis and/or homeostasis of the cell wall play an important role in the resistance to vancomycin observed to DAP strains [39].

Signal transduction mediated by two-component systems (TCSs) YycFG or sensor histidine kinase WalK (also called WalRK) is a regulatory system of two essential components of cell wall synthesis and homeostasis, which has also been implicated in DAP-R and VISA/VSSA cell phenotypes [1, 40]. Members of the two-component regulatory system WalK/WalR that regulate genes are involved in autolysis, biofilm formation and cell wall metabolism [1]. WalK functions as a sensor protein kinase, which is autophosphorylated at a histidine residue and transfers its phosphate group to walk [40]. The impacts of the single substitutions in either WalR or WalK, dramatically change the bacterial cell physiology, with significant reductions in autolytic activity and increases in cell wall thickness linked to the insertion of WalR or WalK alleles from the VISA strain into vancomycin-susceptible S. aureus (VSSA).

The impacts of the single substitutions in either WalR or WalK dramatic changes the cell bacterial physiology, with significant reductions in autolytic activity and increases in cell wall thickness linked to the introduction of the walR or walk allele from the VISA strain into the room fully vancomycin-susceptible S. aureus (VSSA). Vancomycin-intermediate S. aureus (VISA) is generated from vancomycin $S$. aureus sensible by multiple spontaneous mutations in two-component regulatory systems as standing for vancomycin resistance-associated sensor/regulator (VraSR) and inducible by cationic antimicrobial peptides (CAMPs), which could be important for bacterial cells in resisting the harmful effects of CAMPs and possibly other antimicrobial agents [41].

The assessment of the effects of mutations directed at specific targets, which are responsible for the metabolism of the cell wall in S. aureus, indicated that the synergistic mutations (double mutation) might have a more significant effect on the appearance of the VISA phenotype. Recently, it was observed that a deletion mutation in genes of the two-component regulatory system walRK (synonyms: vicRK and yycFG) might result in an increased resistance to vancomycin and appearance of VISA phenotype from the S. aureus LR5P1-V3 strain. On the other hand, LR5P1 strain with double mutation $\left(\right.$ wal $\left.K^{*} c l p P^{*}\right)$ exhibited a thickened cell wall, slow growth and decreased autolytic activity [37]. Similarly, Hu et al. [42], also studying the participation of point mutation in WalK (G223D) gene in S. aureus MW2 (community-acquired methicillin-resistant), reported a decreased expression of genes associated with the cell wall metabolism, decreased autolytic activity and a reduced vancomycin susceptibility. In addition, the electrophoretic mobility shift assay (EMSA) indicated that WalK (G223D)-phosphorylated WalR had a reduced capacity to bind the atlA promoter. The atlA promoter is one of the component potential members of the WalK/WalR regulon and is involved in cell wall metabolism in S. aureus. 
The ATP-dependent Clp protease (ClpP) consists of two heptameric rings and belongs to the family of serine peptidases [43]. ClpP plays an essential role in the degradation of pathogen cell wall but also in the regulation of their virulence [44]. The ClpP components in S. aureus are responsible for initiating physiological adaptive responses against different external pressures, including extreme changes in temperature, osmolarity and oxidative stress [45]. In addition, the ClpP protease presented chaperone-like functions, recognizing and refolding misfolded proteins accumulated or aggregated proteins in the cell, consequently participates in the cell envelope turnover in microbial cells $[46,47]$.

In various pathogenic microorganisms, such as Listeria monocytogenes, Streptococcus pneumoniae and S. aureus, ClpP proteases have been identified in in vivo expression studies as an important virulence factor [44, 48, 49]. Knockout studies of the clpP ATP-dependent gene in S. aureus, followed by global transcriptional analysis using DNA microarray technology, showed that the loss of $c l p P$ leads to complete derepression of transcription of the Cts $R$ gene (or yacG, a polypeptide transcriptional regulator). The $\mathrm{ClpP}$ proteins are regulated by the $C t s R$ heat-shock repressor controlled by HRCA (heat-inducible chaperone, a heat-shock regulon) and a partial derepression of genes involved in response to oxidative stress and DNA repair system SOS response pathway. Moreover, the expression of genes whose products are involved in autolysis of microbial cells was unregulated. This culminated in a dramatic increase in autolysis processes in S. aureus $\triangle c l p P$ mutant [46]. In addition, other observations on the effect of the mutation identified in clpP system in S. aureus LR5P1-V3 strain indicated that deregulation is a new mechanism, which can lead to resistance to vancomycin. It was observed that the LR5P1-V3 mutant, derived from N315LR5P1, exhibited increased resistance to vancomycin, as MIC values rose from 1 to $8 \mu \mathrm{g} / \mathrm{mL}$ [37].

Recently, a genetic evaluation of vancomycin-intermediate S. aureus (VISA) isolated from the same patient at different times tightly corroborates an association of this phenotype with evidence mutations in the novel class of genes, encoding LPXTG motif-containing cell wall-anchoring proteins. These proteins contain an LPXTG sequence motif at their C-terminus, which is a cleavage signal that leads to the covalent binding of the proteins to the cell wall [50]. The presence of covalently bound LPXTG proteins has been shown to contribute to the regular cross-linked structure of the cell wall and anchoring of surface proteins to the peptidoglycan layer [51]. It is hypothesized that the origin of the VISA phenotype may be also related to mutations in the genes encoding LPXTG-associated proteins, leading to an aberrant peptidoglycan structure [50]. This new architecture would provide false binding sites for vancomycin, thus reducing the permeation of this antibiotic in to the cell wall [50]. Mutations evidenced in the anchor domain LPXTG family proteins indicate that the phenotype VISA can have a reduced susceptibility to vancomycin in vivo resulting from spontaneous or induced mutations of little-known character [52].

\section{Mechanisms resistant to $\beta$-lactam antibiotics associated to penicillin- binding protein in Staphylococcus aureus}

With regard to $\beta$-lactam antibiotics, one mechanism-conditioned resistance to these antibiotics, mainly to methicillin and others related drugs, is the expression of penicillin-binding protein 
modified (PBP2s). The PBP2 is a bifunctional protein produced by S. aureus and has binding specificity to $\beta$-lactam antibiotics. This protein presented a functional transpeptidase domain and carboxypeptidase domain; it is directly linked to the cell wall metabolism in bacteria [53]. In S. aureus, both PBPs and (PBP2) are membrane-associated proteins that catalyze the final step of peptidoglycan biosynthesis. The transpeptidase and carboxypeptidase activities of PBPs occur at the D-Ala-D-Ala terminus of a peptidoglycan precursor containing $\mathrm{N}$-acetylglucosamine and N-acetyl-muramic acid L-Ala-D-Glu-L-Lys-D-Ala-D-Ala (pentapeptide chain) [53].

The $\beta$-lactam antibiotics inhibit PBPs by competing with the precursor [undecaprenyl-pyrophosphoryl-MurNAc-(pentapeptide)] for binding to the active site of the enzyme. PBP2s enzymes are associated with decreased bacterial susceptibility to oral cephalosporins and recently, they have been implicated in the emergence of the "MRSA superbugs" [54]. One of the most worrisome evidence on MRSA is related to high mortality rates among infected patients. It is estimated that for MRSA patients, the mortality rate is two to three times higher than for patients infected with S. aureus susceptibly strains [55].

In methicillin-resistant $S$. aureus strains (MRSA), the enzymatic function can be replaced by PBP2 and PBP2s, proteins encoded by the mecA gene, which act as substitutes for the transpeptidase [56]. The mecA gene is present on the staphylococcal cassette chromosome (SCC mec), a genomic island in staphylococci and other Gram-positive bacteria that encode methicillin resistance. Its mobile nature allows it to become widespread among microorganisms [57]. Generally, in susceptible $S$. aureus cells, the vancomycin molecules bind on the C-terminal D-Ala-D-Ala, thereby inhibiting the catalytic reactions of the transglycosylation and transpeptidation, mediated by PBPs. Moreover, PBP2 also plays an important role in the resistance in MRSA; it is related to expression of high-level resistance to vancomycin [58]. This resistance profile is an associated expression of $\operatorname{VanA}$ operon, one complex genic in S. aureus and Enterococcus species designated as vancomycin-resistant enterococci (VRE) [59]. The vanA operon was acquired by VRSA from serving of the transposon $T n 1546$ residing on a conjugal plasmid; possibly, its origin is related to a transfer of the genetic element by association with Enterococcus faecalis in the same niche located in the host [60].

\section{The cell-wall-stress stimulon response in Staphylococcus aureus}

Some antibiotics related to cell wall biogenesis and its stability can influence gene expression and produce a cell-wall-stress stimulon (CWSS) in the S. aureus cells. The oxacillin antibiotics, widely used until recently against $S$. aureus infections, appear to interfere in transcription of genes required for the synthesis of peptidoglycan in the bacterial cells. Analysis of the dynamics of gene expression and proteomic studies using GeneChipsTM approach hypothesized on an upregulation for some proteins and transcription factors involved in cell wall metabolism and response to stress. The same experimental approach that indicated the main influence of amoxicillin during cell exposure is related to increased expression of genes encoding involved in cell wall metabolism, including $p b p B$, Vras and Murz genes. This pattern of transcriptional response can represent the signature of a stimulon cell wall induced in response to antibiotics, which interfere with the synthesis and structure of the cell wall [61]. 
Recently, by using a reporter gene system based on a highly sensitive luciferase fused with tcaA and sa0908 genes, it was demonstrated that gene expression in S. aureus occurs in different kinetic patterns [62]. The $t c a A$ and $s a 0908$ genes relate to encoding a membrane protein related to signature resistance to glycopeptide antibiotics and encoding protein for the cell envelope (member of the LytR-CpsA-Psr family), respectively. These patterns were determined from observations of the fluorescence intensity during growth of $S$. aureus (susceptible strain BB255) in the presence of various antibiotics at subinhibitory and inhibitory concentrations. From their results, it was possible to establish that all drugs tested induced the CWSS and that the induction patterns varied according to the drug. One of the hypotheses raised in this study would be that the standard kinetics observed could perhaps be related to the specificity or antibiotic mechanism action. These findings are based on fluorescence results obtained for different drug groups such as tunicamycin, flavomycin, oxacillin and fosfomycin, which presented high levels of maximal induction (RLU > 40,000); and daptomycin and lysostaphin exhibiting fluorescence indices below 10,000. Moreover, some antibiotics such as fosfomycin and D-cycloserine showed a lag-phase induction for all tested concentrations of about 30 and $10 \mathrm{~min}$, respectively. Possibly, these antibiotics act in the early stages of peptidoglycan synthesis, which could be linked to delays in the induction CWSS in the S. aureus.

\section{Conclusion}

Multiple mechanisms contribute to the increased antimicrobial resistance in S. aureus, culminating in a more robust cellular adaptation against antibiotics that act on the synthesis of components of the cell envelope. These multiple mechanisms of adaptation in S. aureus include heteroresistance or mutational events, make the bacteria to evolve from a susceptible strain in to a resistant clone; thus significantly interfering with the bacterial response to antibiotics. Finally, further knowledge of these mechanisms may considerably impact the development of new drugs designed to specific targets in the microbial cell.

\section{Author details}

Andrea de Souza Monteiro ${ }^{1 *}$, Wallace Ribeiro Nunes $\mathrm{Neto}^{1}$, Aleff Ricardo Santos Mendes ${ }^{1}$, Bruna Lorrana dos Santos Pinto ${ }^{1}$, Luís Cláudio Nascimento da Silva ${ }^{2}$ and Gabriella Freitas Ferreira $^{3}$

*Address all correspondence to: andreasmont@gmail.com

1 Laboratório de Microbiologia, Universidade CEUMA, São Luis, Maranhão, Brazil

2 Laboratório de Prospecção Molecular, Universidade CEUMA, São Luis, Maranhão, Brazil

3 Departamento de Farmácia, Universidade Federal de Juiz de Fora, Governador Valadares, Brazil 


\section{References}

[1] V. Cafiso, et al. Modulating activity of vancomycin and daptomycin on the expression of autolysis cell-wall turnover and membrane charge genes in hVISA and VISA strains. PLoS One. 2012;7(1):e29573. DOI: 10.1371/journal.pone.0029573.

[2] S.D. Taylor, M. Palmer. The action mechanism of daptomycin. Bioorganic \& Medicinal Chemistry. 2016; 24 (24):6253-6268. DOI: 10.1016/j.bmc.2016.05.052.

[3] C.L. Moore, et al. Daptomycin versus vancomycin for bloodstream infections due to methicillin-resistant Staphylococcus aureus with a high vancomycin minimum inhibitory concentration: a case-control study. Clinical Infectious Diseases. 2012;54(1):51-58. DOI: 10.1093/cid/cir764.

[4] F.P Tally, et al. Daptomycin: a novel agent for gram-positive infections. Expert Opinion on Investigational Drugs. 1999;8(8):1223-1238. DOI: 10.1517/13543784.8.8.1223.

[5] J.K. Hobbs, et al. Consequences of daptomycin-mediated membrane damage in Staphylococcus aureus. Journal of Antimicrobial Chemotherapy. 2008;62(5):1003-1008. DOI: $10.1093 /$ jac/dkn321.

[6] P. Canepari, et al. Lipoteichoic acid as a new target for activity of antibiotics: mode of action of daptomycin (LY146032). Antimicrobial Agents and Chemotherapy. 1990;34(6):1220-1226. DOI: 10.1128/AAC.34.6.1220.

[7] N.E. Allen, J.N. Hobbs, W.E. Alborn Jr. Inhibition of peptidoglycan biosynthesis in gram-positive bacteria by LY146032. Antimicrobial Agents and Chemotherapy. 1987;31(7):1093-1099. DOI: 10.1128/AAC.31.7.1093.

[8] J.K. Muraiha, et al. Oligomerization of daptomycin on membranes. Biochimica et Biophysica Acta (BBA)-Biomembranes. 2011;1808(4):1154-1160. DOI: 10.1016/j. bbamem.2011.01.001.

[9] R.D. Süssmuth, W. Wohlleben. The biosynthesis of glycopeptide antibiotics - a model for complex, non-ribosomally synthesized, peptidic secondary metabolites. Applied Microbiology and Biotechnology. 2004;63(4):344-350. DOI: 10.1007/s00253-003-1443-z.

[10] H. Jeong, et al. Genome sequence of the vancomycin-producing amycolatopsis orientalis subsp. orientalis strain KCTC 9412T. Genome Announcements. 2013;1(3):e00408-e00413. DOI: 10.1128/genomeA.00408-13.

[11] S.T. Micek. Alternatives to vancomycin for the treatment of methicillin-resistant Staphylococcus aureus infections. Clinical Infectious Diseases. 2007;45(3):S184-S190. DOI: $10.1086 / 519471$.

[12] G.F. Ferreira, et al. Heteroresistance to itraconazole alters the morphology and increases the virulence of cryptococcus gattii. Antimicrobial Agents and Chemotherapy. 2015;59(8):4600-4609. DOI: 10.1128/AAC.00466-15. 
[13] S. Deresinski. Vancomycin heteroresistance and methicillin-resistant Staphylococcus aureus. The Journal of Infectious Diseases. 2009;199(5):605-609. DOI: 10.1086/596630.

[14] C. Liu, H.F. Chambers. Staphylococcus aureus with heterogeneous resistance to vancomycin: epidemiology, clinical significance and critical assessment of diagnostic methods. Antimicrobial Agents and Chemotherapy. 2003;47(10):3040-3045. DOI: 10.1128/ AAC.47.10.3040-3045.2003.

[15] Y.Maor, et al. Clinical features of heteroresistant vancomycin-intermediate Staphylococcus aureus bacteremia versus those of methicillin-resistant $S$. aureus bacteremia. The Journal of Infectious Diseases. 2009;199(5):619-624. DOI: 10.1086/596629.

[16] G. Sakoulas, et al. Adaptation of methicillin-resistant Staphylococcus aureus in the face of vancomycin therapy. Clinical Infectious Diseases. 2006;42(1):S40-S50. DOI: 10.1086/491713.

[17] L. Cui, et al. Novel mechanism of antibiotic resistance originating in vancomycin-intermediate Staphylococcus aureus. Antimicrobial Agents and Chemotherapy. 2006;50(2):428438. DOI: 10.1128/AAC.50.2.428-438.2006.

[18] F. Bert, et al. Prevalence, molecular epidemiology and clinical significance of heterogeneous glycopeptide-intermediate Staphylococcus aureus in liver transplant recipients. Journal of Clinical Microbiology. 2003;41(11):5147-5152. DOI: 10.1128/ JCM.41.11.5147-5152.2003.

[19] A. Capone, et al. In vivo development of daptomycin resistance in vancomycin-susceptible methicillin-resistant Staphylococcus aureus severe infections previously treated with glycopeptides. European Journal of Clinical Microbiology \& Infectious Diseases. 2016;35(4):625-631. DOI: 10.1007/s10096-016-2581-4.

[20] G. Sakoulas, et al. Human cathelicidin LL-37 resistance and increased daptomycin MIC in methicillin-resistant Staphylococcus aureus strain USA600 (ST45) are associated with increased mortality in a hospital setting. Journal of Clinical Microbiology. 2014;52(6):2172-2174. DOI: 10.1128/JCM.00189-14.

[21] B.P. Howden, et al. Reduced vancomycin susceptibility in Staphylococcus aureus, including vancomycin-intermediate and heterogeneous vancomycin-intermediate strains: resistance mechanisms, laboratory detection and clinical implications. Clinical Microbiology Reviews. 2010;23(1):99-139. DOI: 10.1128/CMR.00042-09.

[22] N.N. Mishra, et al. In vitro cross-resistance to daptomycin and host defense cationic antimicrobial peptides in clinical methicillin-resistant Staphylococcus aureus isolates. Antimicrobial Agents and Chemotherapy. 2011;55(9):4012-4018. DOI: 10.1128/AAC.00223-11.

[23] T.T. Tran, et al. Mechanisms of drug resistance: daptomycin resistance. Annals the New York Academy of Sciences. 2015;1354:32-53. DOI: 10.1111/nyas.12948.

[24] L. Dortet, et al. In vivo acquired daptomycin resistance during treatment of methicillinresistant Staphylococcus aureus endocarditis. International Journal of Infectious Diseases. 2013;17(11):e1076-e1077. DOI: 10.1016/j.ijid.2013.02.019. 
[25] K. Julian, et al. Characterization of a daptomycin-nonsusceptible vancomycin-intermediate Staphylococcus aureus strain in a patient with endocarditis. Antimicrobial Agents and Chemotherapy. 2007;51(9):3445-3448. DOI: 10.1128/AAC.00559-07.

[26] S.J. van Hal, et al. Emergence of daptomycin resistance following vancomycin-unresponsive Staphylococcus aureus bacteraemia in a daptomycin-naïve patient-a review of the literature. European Journal of Clinical Microbiology \& Infectious Diseases. 2011;30(5):603-610. DOI: 10.1007/s10096-010-1128-3.

[27] J.B. Patel, et al. An association between reduced susceptibility to daptomycin and reduced susceptibility to vancomycin in Staphylococcus aureus. Clinical Infectious Diseases. 2006;42(11):1652-1653. DOI: 10.1086/504084.

[28] Y. Oku, et al. Characterization of the Staphylococcus aureus mprF gene, involved in lysinylation of phosphatidylglycerol. Microbiology. 2004;150(1):45-51. DOI: 10.1099/mic.0.26706-0.

[29] C.M. Ernst, et al. The lipid-modifying multiple peptide resistance factor is an oligomer consisting of distinct interacting synthase and flippase subunits. MBio. 2015;6(1):e02340 e02414. DOI: 10.1128/mBio.02340-14.

[30] J. Andrä, et al. Multiple peptide resistance factor (MprF)-mediated resistance of Staphylococcus aureus against antimicrobial peptides coincides with a modulated peptide interaction with artificial membranes comprising Lysyl-phosphatidylglycerol. The Journal of Biological Chemistry. 2011;286:18692-18700. DOI: 10.1074/jbc.M111.226886.

[31] C.M. Ernst, et al. The bacterial defensin resistance protein MprF consists of separable domains for lipid lysinylation and antimicrobial peptide repulsion. PLoS Pathogens. 2009;5(11):e1000660. DOI: 10.1371/journal.ppat.1000660.

[32] A. Peschel, et al. Staphylococcus aureus resistance to human defensins and evasion of neutrophil killing via the novel virulence factor mprf is based on modification of membrane lipids with L-lysine. JEM. 2001;193(9):1067. DOI: 10.1084/jem.193.9.1067.

[33] C.J. Slavetinsky, A. Peschel, C.M. Ernst. Alanyl-phosphatidylglycerol and lysyl-phosphatidylglycerol are translocated by the same MprF flippases and have similar capacities to protect against the antibiotic daptomycin in Staphylococcus aureus. Antimicrobial Agents and Chemotherapy. 2012;56(7):3492-3497. DOI: 10.1128/AAC.00370-12.

[34] R.H Baltz. Daptomycin: mechanisms of action and resistance and biosynthetic engineering. Current Opinion in Chemical Biology. 2009;13(2):144-151. DOI: 10.1016/j. cbpa.2009.02.031.

[35] S.J. Yang, et al. Causal role of single nucleotide polymorphisms within the mprF gene of Staphylococcus aureus in daptomycin resistance. Antimicrobial Agents and Chemotherapy. 2013;57(11):5658-5664. DOI: 10.1128/AAC.01184-13.

[36] A. Jansen, et al. Role of insertion elements and yycFG in the development of decreased susceptibility to vancomycin in Staphylococcus aureus. International Journal of Medical Microbiology. 2007;297(4):205-215. DOI: 10.1016/j.ijmm.2007.02.002. 
[37] M. Shoji, et al. WalK and clpP mutations confer reduced vancomycin susceptibility in Staphylococcus aureus. Antimicrobial Agents Chemotherapy. 2011;55(8):3870-3881. DOI: 10.1128/AAC.01563-10.

[38] A. Muthaiyan, et al. Transcriptional profiling reveals that daptomycin induces the Staphylococcus aureus cell wall stress stimulon and genes responsive to membrane depolarization. Antimicrobial Agents and Chemotherapy. 2008;52(3):980-990. DOI: 10.1128/ AAC.01121-07.

[39] A. Fischer, et al. Daptomycin resistance mechanisms in clinically derived Staphylococcus aureus strains assessed by a combined transcriptomics and proteomics approach. Journal of Antimicrobial Chemotherapy. 2011;66(8):1696-1711. DOI: 10.1093/jac/dkr195.

[40] Q. Ji, et al. Structure and mechanism of the essential two-component signal-transduction system WalKR in Staphylococcus aureus. Nature Communications. 2016;7(11000). DOI: 10.1038/ncomms11000.

[41] S. Dubrac, T. Msadek. Identification of genes controlled by the essential YycG/YycF twocomponent system of Staphylococcus aureus. Journal of Bacteriology. 2004;186(4):11751181. DOI: 10.1128/JB.186.4.1175-1181.2004.

[42] J. Hu, et al. Mechanism of reduced vancomycin susceptibility conferred by walk mutation in community-acquired methicillin-resistant Staphylococcus aureus strain MW2. Antimicrobial Agents and Chemotherapy. 2014;59(2):1352-1355. DOI: 10.1128/ AAC.04290-14.

[43] M.R. Maurizi. Clp P represents a unique family of serine proteases. The Journal of Biological Chemistry. 1990;265(21):12546-12552. http://www.jbc.org/content/265/21/ 12546.long.

[44] A.J. Farrand, et al. Regulation of host hemoglobin binding by the Staphylococcus aureus Clp proteolytic system. Journal of Bacteriology. 2013;195(22):5041-5050. DOI: 10.1128/ JB.00505-13.

[45] D. Frees, et al. Alternative roles of ClpX and ClpP in Staphylococcus aureus stress tolerance and virulence. Molecular Microbiology. 2003;48(6):1565-1578. DOI: 10.1046/j.1365-2958.2003.03524.x.

[46] A. Michel, et al. Global regulatory impact of ClpP protease of Staphylococcus aureus on regulons involved in virulence, oxidative stress response, autolysis and DNA repair. Journal of Bacteriology. 2006;188(16):5783-5796. DOI: 10.1128/JB.00074-06.

[47] A. Wawrzynow, et al. The ClpX heat-shock protein of Escherichia coli, the ATP-dependent substrate specificity component of the ClpP-ClpX protease, is a novel molecular chaperone. The EMBO Journal. 1995;14(9):1867-1877. http://www.ncbi.nlm.nih.gov/pmc/ articles/PMC398286/. 
[48] G.T. Robertson, et al. Global transcriptional analysis of clpP mutations of type 2 Streptococcus pneumoniae and their effects on physiology and virulence. Journal of Bacteriology. 2002;184(13):3508-3520. DOI: 10.1128/JB.184.13.3508-3520.2002.

[49] O. Gaillot, et al. The ClpP serine protease is essential for the intracellular parasitism and virulence of Listeria monocytogenes. Molecular Microbiology. 2000;35(6):1286-1294. DOI: 10.1046/j.1365-2958.2000.01773.x.

[50] L. Rishishwar, C.S. Kraft, I. King Jordan. Population genomics of reduced vancomycin susceptibility in Staphylococcus aureus. mSphere. 2016;1(4):e00094-e00116. DOI: 10.1128/ mSphere.00094-16.

[51] S.K. Mazmanian, et al. Staphylococcus aureus sortase, an enzyme that anchors surface proteins to the cell wall. Science. 1999;285(5428):760-763. DOI: 10.1126/ science.285.5428.760.

[52] H. Ton-That, O. Schneewind. Anchor structure of Staphylococcal surface proteins. The Journal of Biological Chemistry. 1999;274(34):24316-24320. http://www.jbc.org/content/274/34/24316.full.pdf.

[53] V. Navratna, et al. Molecular basis for the role of Staphylococcus aureus penicillin binding protein 4 in antimicrobial resistance. Journal of Bacteriology. 2010;192(1):134-144. DOI: 10.1128/JB.00822-09.

[54] A. Ojha Kshetry, et al. Minimum inhibitory concentration of vancomycin to methicillin resistant Staphylococcus aureus isolated from different clinical samples at a tertiary care hospital in Nepal. Antimicrobial Resistance Infection Control. 2016;5(27). DOI: 10.1186/ s13756-016-0126-3.

[55] R. Köck, et al. Methicillin-resistant Staphylococcus aureus (MRSA): burden of disease and control challenges in Europe. European Surveillance. 2010;15(41): ):pii=19688. http:// www.eurosurveillance.org/images/dynamic/ee/v15n41/art19688.pdf.

[56] K. Hiramatsu, et al. The emergence and evolution of methicillin-resistant Staphylococcus aureus. Trends in Microbiology. 2001;9(10):486-493. DOI: 10.1016/ S0966-842X(01)02175-8.

[57] Y. Katayama, et al. PBP 2a mutations producing very-high-level resistance to betalactams. Antimicrobial Agents and Chemotherapy. 2004;48(2):453-459. DOI: 10.1128/ AAC.48.2.453-459.2004.

[58] B.J. Werth, et al. Novel combinations of vancomycin plus ceftaroline or oxacillin against methicillin-resistant vancomycin-intermediate Staphylococcus aureus (VISA) and heterogeneous VISA. Antimicrobial Agents and Chemotherapy. 2013;57(5):2376-2379. DOI: 10.1128/AAC.02354-12. 
[59] B. Périchon, P. Courvalin. VanA-type vancomycin-resistant Staphylococcus aureus. Antimicrobial Agents and Chemotherapy. 2009;53(11):4580-4587. DOI: 10.1128/AAC. 00346-09.

[60] L.M. Weigel, et al. Genetic analysis of a high-level vancomycin-resistant isolate of Staphylococcus aureus. Science. 2003;302(5650):1569-1571. DOI:10.1126/science.1090956.

[61] S. Utaida, et al. Genome-wide transcriptional profiling of the response of Staphylococcus aureus to cell-wall-active antibiotics reveals a cell-wall-stress stimulon. Microbiology. 2003;149(10):2719-2732. DOI: 10.1099/mic.0.26426-0.

[62] V. Dengler, et al. Induction kinetics of the Staphylococcus aureus cell wall stress stimulon in response to different cell wall active antibiotics. BMC Microbiology. 2011;11(16). DOI: 10.1186/1471-2180-11-16. 